\section{Panorama histórico del desarrollo de la energía nu- cleoeléctrica en Argentina}

Resumen: Argentina es uno de los pocos países que inició tempranamente, en 1949, una actividad nuclear relevante con fines pacíficos, desarrollando capacidades científicas y tecnológicas propias en este campo. A pesar de un recorrido complejo, caracterizado por políticas estatales de impulso y retroceso, el desarrollo nuclear nacional ha logrado alcanzar una posición de liderazgo en la región. Entre sus logros más destacados se puede mencionar la construcción de reactores de investigación, la obtención y producción de combustible nuclear para su operación y el dominio del ciclo de combustible, la construcción de centrales nucleares para la generación de energía eléctrica, el desarrollo de la medicina nuclear, la creación de empresas de base tecnológica como INVAP S.E. y el desarrollo, con el Proyecto CAREM, de reactores nucleares modulares de baja potencia íntegramente diseñados y construidos en el país. En este trabajo se brinda un panorama histórico de la evolución de las actividades nucleares en nuestro país y de las políticas que permitieron consolidar una trama productiva local con capacidades de generar desarrollos tecnológicos autónomos.

Palabras clave: política nuclear, energía nucleoeléctrica, Proyecto CAREM, Desarrollo autónomo

\section{Historical overview of the development of nuclear power in Argentina}

Summary: Argentina is one of the few countries that began early, in 1949, a relevant nuclear activity for peaceful purposes, developing its own scientific and technological capabilities in this field. Despite a complex journey, characterized by state policies of push and pull, national nuclear development has managed to achieve a leadership position in the region. Among his most outstanding achievements we can mention the construction of research reactors, the obtaining and production of nuclear fuel for its operation and the control of the fuel cycle, the construction of nuclear power plants for the generation of electrical energy, the development of medicine nuclear, the creation of technology-based companies such as INVAP SE and the development, with the CAREM Project, of modular low-power nuclear reactors fully designed and built in the country. This paper provides a historical overview of the evolution of nuclear activities in our country and of the policies that made it possible to consolidate a local productive network with capacities to generate autonomous technological developments.

Keywords: nuclear policy, nuclear power, CAREM Project, Autonomous development

\section{Panorama histórico do desenvolvimento da energia nuclear na Argentina}

Resumo:A Argentina é um dos poucos países que iniciou cedo, em 1949, uma atividade nuclear relevante com fins pacíficos, desenvolvendo suas próprias capacidades científicas e tecnológicas nesse campo. Apesar de uma jornada complexa, caracterizada por políticas estaduais de impulso e retrocesso, o desenvolvimento nuclear nacional conseguiu alcançar uma posição de liderança na região. Entre suas realizações mais destacadas, podemos citar a construção de reatores de pesquisa, a obtenção e produção de combustível nuclear para seu funcionamento e o controle do ciclo do combustível, a construção de usinas nucleares para a geração de energia elétrica, o desenvolvimento da medicina nuclear , a criação de empresas de base tecnológica, como a INVAP SE, e o desenvolvimento, com o Projeto CAREM, de reatores nucleares modulares de baixa potência totalmente projetados e construídos no país. Este artigo oferece um panorama histórico da evolução da atividade nuclear em nosso país e das políticas que possibilitaram a consolidação de uma rede produtiva local com capacidade de gerar desenvolvimento tecnológico autônomo.

Palavras-chave: política nuclear, energia nuclear, Projeto CAREM, Desenvolvimento autônomo.

\section{Ciencia Tecnología y Política Año $4 \mathrm{~N}^{\circ} 7$ Noviembre 2021}

Oscar Ramirez, Jorge Nicolini, Marcelo Neuman, Marcelo

Fernandéz y Jorge Malco.

Universidad Nacional de

General Sarmiento

oramirez@campus.ungs.edu.ar

Año $4 \mathrm{~N}^{\circ} 7$ Noviembre 2021

Fecha de recibido: $12 / 07 / 2021$

Fecha de aprobado: 29/10/2021

https://doi.org/10.24215/26183188e068

https://revistas.unlp.edu.ar/CTyP

ISSN 2618-3188

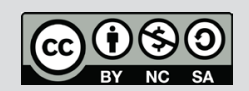

Esta obra está bajo licencia Creative Commons Atribución-NoComercial-Compartirlgual 4.0 Internacional http://creativecommons.org/licenses/bync-sa/4.0/deed.es_AR 


\section{Panorama histórico del desarrollo de la energía nucleoeléctrica en Argentina}

Oscar Ramirez, Jorge Nicolini,

Marcelo Neuman, Marcelo Fernandéz

y Jorge Malco.

Universidad Nacional de General Sarmiento oramirez@campus.ungs.edu.ar

Resumen: Argentina es uno de los pocos países que inició tempranamente, en 1949, una actividad nuclear relevante con fines pacíficos, desarrollando capacidades científicas y tecnológicas propias en este campo. A pesar de un recorrido complejo, caracterizado por políticas estatales de impulso y retroceso, el desarrollo nuclear nacional ha logrado alcanzar una posición de liderazgo en la región. Entre sus logros más destacados se puede mencionar la construcción de reactores de investigación, la obtención y producción de combustible nuclear para su operación y el dominio del ciclo de combustible, la construcción de centrales nucleares para la generación de energía eléctrica, el desarrollo de la medicina nuclear, la creación de empresas de base tecnológica como INVAP S.E. y el desarrollo, con el Proyecto CAREM, de reactores nucleares modulares de baja potencia íntegramente diseñados y construidos en el país. En este trabajo se brinda un panorama histórico de la evolución de las actividades nucleares en nuestro país y de las políticas que permitieron consolidar una trama productiva local con capacidades de generar desarrollos tecnológicos autónomos.

\section{Inicio de las actividades nucleares en Argentina}

Argentina es uno de los pocos países que inició tempranamente una actividad nuclear relevante desarrollando capacidades científicas y tecnológicas endógenas, tanto en el área nuclear específica como en las tecnologías asociadas. Las actividades nucleares en el país se iniciaron en junio de 1949 con el "Proyecto Huemul", la Planta Experimental de Altas Temperaturas emplazada en la isla Huemul, en las afueras de Bariloche. Este proyecto fue dirigido por el físico austríaco Ronald Richter (Sábato, 1968). El 31 de mayo de 1950 se crea la Comisión Nacional de Energía Atómica (CNEA), quedando a su cargo el coronel Enrique P. González, siendo en sus inicios una especie de comisión de notables que tenía entre sus objetivos controlar la efectividad del proyecto Huemul.

En el año 1951 se crea la Dirección Nacional de Energía Atómica (DNEA), dependiente del Ministerio de Asuntos Técnicos. Entre sus funciones tuvo como prioridad el entrenamiento de científicos y técnicos locales. En febrero de 1952, el presidente Perón cesa en sus funciones a González y pone al frente de la CNEA al capitán de fragata Pedro Iraolagoitía. Luego de un período de inocultables fracasos, el 11 de noviembre de ese año Iraolagoitía ingresa en la isla Huemul en una operación militar y clausura el Proyecto Huemul (Hurtado de Mendoza, 2014).

Con el derrocamiento de Perón en el año 1955, la original CNEA fue cerrada y asimilada a la DNEA, 
| Ciencia, Tecnología y Política | Año 4 | Nº7 | e068 | Noviembre 2021 | ISSN 2618-3188 | www.revistas.unlp.edu.ar/CTyP |

llamándose definitivamente CNEA y manteniendo la dependencia del Poder Ejecutivo (Hurtado de Mendoza, 2005). En la década del '50 las actividades del organismo se enfocan en la formación de recursos humanos y el desarrollo de infraestructura. En 1955 se inaugura el Centro Atómico Bariloche (CAB) y se crea el Instituto Balseiro en asociación con la Universidad de Cuyo y distintas carreras universitarias relacionadas con la temática nuclear, también comienza a realizarse la extracción del mineral de uranio (Nevia Vera y Colombo, 2014). En 1958 se inaugura el Centro Atómico Constituyentes (CAC), y se inicia el proceso de producción de radioisótopos para distintas aplicaciones (Colombo, Guglielminotti y Nevia Vera, 2017). También ese año empieza a operar el reactor RA1 en el CAC, construido 100\% en Argentina, pasando a ser el primer reactor experimental de América Latina. Por otra parte, desde 1957, la CNEA había empezado a diseñar y fabricar los elementos combustibles para los reactores de investigación que fueron poniéndose en funcionamiento (Consejo Argentino para las Relaciones Internacionales, 1999).

En la década del '60 se da impulso a los trabajos de prospección y exploración de minerales de uranio, construyéndose en la provincia de Salta una planta de lixiviación en pilas, que empieza a operar en 1961.

\section{Etapa nucleoeléctrica}

En el año 1964 la CNEA propone al Poder Ejecutivo Nacional estudiar la factibilidad de la construcción de una central nuclear de generación eléctrica. Como consecuencia de este pedido, el Poder Ejecutivo encomienda a este organismo realizar un estudio de pre inversión para instalar una central en el área del Gran Buenos Aires-Litoral (Sábato, 1968).

Por otra parte, en 1965 comienza a funcionar en Mendoza la primera planta de producción de con- centrado de uranio. De este modo para fines de la década del '60 ya se encontraba afianzada la explotación de recursos de uranio con vistas a proveer de combustible a una futura central nucleoeléctrica.

En 1967 se inaugura el reactor RA3 dedicado a la producción de radioisótopos para uso médico e industrial y en 1971 empieza a funcionar la respectiva planta de producción de este insumo. Ambos proyectos fueron diseñados y desarrollados por CNEA e instalados en el Centro Atómico Ezeiza (CAE).

En 1967, además, se llamó a licitación internacional para la construcción llave en mano de la primera central nuclear del país, la que se adjudicó a la empresa Siemens AG, entrando en operación comercial en junio de 1974. La potencia neta instalada fue de $330 \mathrm{MW}$ utilizando un modelo de reactor de recipiente a presión y agua pesada como elemento moderador y refrigerante. El combustible elegido fue uranio natural, siendo emplazada en Atucha (partido de Zárate), provincia de Buenos Aires.

La segunda central nucleoeléctrica del país se instaló en la ribera sur del Embalse del Río Tercero. Su construcción se inició el 7 de mayo de 1974 y el 20 de enero de 1984 comenzó su operación comercial. Es del tipo CANDU con un reactor de potencia del tipo de tubos de presión de $600 \mathrm{MW}$, también a base de uranio natural como combustible y agua pesada como elemento moderador y refrigerante.

La estrategia de utilizar uranio natural de producción nacional en estas dos primeras centrales nucleares se debió a la dificultad de alcanzar la tecnología de enriquecimiento de uranio por las restricciones existentes a nivel internacional. Esto permitió independizarse del virtual oligopolio asociado con la provisión de uranio enriquecido utilizado en los reactores del tipo de agua a presión y lograr de esta manera el dominio total del ciclo del 
combustible. Por otra parte, se optó por el agua pesada como elemento moderador y refrigerante pues se tenía prevista su producción en el país a corto plazo (De Dicco, 2015).

Durante la dictadura militar (1976-1983) los planes nucleares se intensificaron, en medio de un contexto macroeconómico que promovía una desindustrialización masiva. Esta circunstancia se explica en gran parte por el lugar estratégico que ocupaba la industria nuclear en el pensamiento de las fuerzas armadas (Hurtado de Mendoza, 2009). Es así como en 1979 se realiza el llamado a licitación para la construcción de una tercera central nuclear de $700 \mathrm{MW}$ de potencia, conocida como Central Nuclear Atucha II (CNAII). Al igual que en las centrales que ya estaban en operación ésta también utilizaría uranio natural y agua pesada. También se asumía el compromiso de contar con una planta de agua pesada que abasteciera las centrales proyectadas en el plan nuclear. Entre los factores tenidos en cuenta para la adjudicación, fue muy valorada la participación nacional en todos los rubros del proyecto (Quilici, 2008). Para su construcción cinco empresas presentaron ofertas, aprobándose la propuesta de la empresa Kraftwerk Union AG (KWU) subsidiaria de Siemens AG, que consistía en una central de 692 MW de potencia neta y la creación con la firma alemana de una empresa de capitales mixtos de ingeniería para su construcción (De Dicco, 2015). Se considera que en el resultado de la licitación influyeron las gestiones de Alemania para que la empresa Sulzer Brothers de Suiza instalara llave en mano una planta industrial de agua pesada (Quilici, 2008). Dicha empresa inició la construcción de la misma en 1980 en Arroyito, Pcia. de Neuquén, inaugurándose en 1994, con una producción anual de 200 toneladas, previamente había entrado en operación una planta piloto construida en Atucha en 1984 (Consejo Argentino para las Relaciones Internacionales, 1999).
En 1980 se constituye la Empresa Nuclear Argentina de Centrales Eléctricas S.A. (ENACE) de la cual CNEA poseía el 75\% y KWU el 25\%. Esta empresa pasó a dirigir el proyecto de construcción de la CNAll, quedando a su cargo la obra civil e industrial, licitaciones, supervisión de contratos y el control de calidad. El objetivo era que ENACE capacitara a la industria nacional en el diseño y construcción de plantas nucleares (De Dicco, 2015). A partir de 1983, la obra sufrió innumerables retrasos a consecuencia de limitaciones presupuestarias. En 1989, con la implantación del neoliberalismo en el país (Bisang, 1995), la política nuclear sufrió fuertes recortes presupuestarios que llevaron a la paralización de la CNA II y la pérdida de personal científico y técnico (Nevia Vera y Colombo, 2014).

Con el fin del modelo neoliberal de los '90 y la llegada a la presidencia de Néstor Kirchner en el 2003, se vuelve a impulsar la reindustrialización del país y se plantea la necesidad de diversificar la matriz energética. Esto propició el resurgimiento de la actividad nuclear. En 2004 se diseña un plan de reactivación nuclear con el objetivo de reimpulsar la generación eléctrica y las aplicaciones en el campo de la salud y la industria, incluyéndose como ítem relevante la terminación de la CNAll (Colombo, Guglielminotti y Nevia Vera, 2017), cuyas obras se reinician en 2006. Su reactivación requirió la formación de miles de profesionales, técnicos y oficiales calificados y el financiamiento del Estado para la fabricación de componentes críticos. Entre diciembre de 2006 y septiembre de 2011 se completó la obra civil, se ejecutaron los montajes electromecánicos pendientes (en el orden del 50\%) y se construyó el 100\% de la infraestructura complementaria, participando con el 88\% la industria nacional. El 3 de junio de 2014 se logra la primera criticidad del reactor y el 27 de junio la sincronización con la red eléctrica, alcanzándose el $100 \%$ de potencia el 18 de febrero de 2015 (De Dicco, 2015). 


\section{Dominio del ciclo de combustible nuclear}

A pesar de que la central nuclear Atucha I (CNAl) fue comprada con la carga de los elementos combustibles (EECC), la CNEA siempre tuvo prevista la fabricación de los mismos en el país. Por Decreto del PEN en diciembre de 1981 se creó la empresa Combustibles Nucleares Argentinos (CONUAR) con el objetivo de encargarse de la fabricación del combustible nuclear que abastecería a las centrales. Para ello se utilizó el formato de sociedad anónima integrada por CNEA y el holding Pérez Companc. La inauguración de la planta productiva se produjo el 2 de abril de 1982, iniciando de esta forma la provisión de combustible a la CNA I.

Con el objetivo de suministrar EECC a la Central Nuclear Embalse (CNE), se genera el proyecto "Suministro de Combustibles para Embalse" (SUCOEM). La producción de estos elementos bajo la responsabilidad de CNEA, comienza en diciembre de 1983 y se extiende fabricando 3000 EECC que reemplazaron gradualmente los originales canadienses. En 1988 CONUAR se hace cargo de la línea de producción que abastece a la CNE (Quilici, 2010). En cuanto a la CNA II, entre diciembre de 2012 y febrero de 2016 se cargaron sus 451 elementos combustibles fabricados por CONUAR (De Dicco, 2015).

Otro desafío importante en el camino de dominar la tecnología para la elaboración de los EECC, fue desarrollar la capacidad de fabricación de los elementos estructurales que los conforman. Así, CNEA en 1974 crea el "Programa Tecnología de Circonio y sus Aleaciones", dando con esto origen al desarrollo de esa tecnología a través de la Planta Piloto de Fabricación de Aleaciones Especiales (PPFAE) emplazada en el CAE. El proyecto para la implementación industrial se realiza a través de la Fábrica de Aleaciones Especiales (FAE) también ubicada en el CAE (Quilici, 2010) y creada el 30 de junio de 1986. El capital accionario de
FAE S.A. se constituye con un $68 \%$ de CONUAR y un $32 \%$ de CNEA. Desde ese año fabrica, a escala industrial, vainas y otros componentes estructurales, que luego son integrados por CONUAR junto a las pastillas de uranio para la elaboración de los EECC (Quilici, 2008).

Con el objetivo de dominar el ciclo del combustible nuclear y lograr independencia de proveedores extranjeros, en 1979 la CNEA da inicio al proyecto de construir una planta de producción de polvo de dióxido de uranio de pureza nuclear grado cerámico. Para ello adopta la opción de comprar a la empresa alemana RBU una planta "llave en mano" instalándola en el barrio Alta Córdoba de la Ciudad de Córdoba (Quilici, 2010). Esta planta empieza a producir en noviembre de 1982 dióxido de uranio (UO2) a escala industrial con el objetivo de proveerlo a CONUAR, para fabricar las pastillas de uranio, tanto natural como levemente enriquecido, y los EECC para ser utilizados en los reactores de las centrales. Con estos desarrollos Argentina pasó a formar parte de los diez países que cuentan con la capacidad para diseñar y producir combustibles nucleares.

Durante el período de gobierno de Carlos Menem (1989-1999), se crea en 1997 la empresa Dioxitek SA, que pasa a tomar el control de la planta de Córdoba, otorgándole a CNEA el 99\% de las acciones y a Nuclear Mendoza S.E. el 1\%. Hoy en día, CNEA posee el 48\% de las acciones, la Secretaría de Energía de la Nación el 51\%, manteniendo Nuclear Mendoza el 1\% restante.

Posteriormente, en el período de gobierno de Néstor Kirchner y como parte del proceso de reactivación del Plan Nuclear, se pone en marcha un proyecto para aumentar la producción de dióxido de uranio, dentro del cual se planificó el traslado de la planta a la provincia de Formosa, con el objetivo de aumentar su producción a 230 toneladas al año. Dicha planta aún se encuentra en proceso de construcción. 
| Ciencia, Tecnología y Política | Año 4 | Nº7 | e068 | Noviembre 2021 | ISSN 2618-3188 | www.revistas.unlp.edu.ar/CTyP |

\section{INVAP y el proyecto Pilcaniyeu de enriqueci- miento de uranio}

En 1971, un grupo de investigadores del Centro Atómico Bariloche y de la CNEA creó el Programa de Investigaciones Aplicadas (PIA). El principal objetivo del PIA era utilizar los conocimientos disponibles en la CNEA y las capacidades acumuladas en el Centro Atómico Bariloche para contribuir al desarrollo de tecnologías para la industria local.

La dinámica del Programa mostró rápidamente que cuanto más se ampliaba la cantidad de contrataciones, mayor era la dificultad administrativa y de gerenciamiento de los emprendimientos que se realizaban. En parte por estas dificultades, hacia fines de 1975, los integrantes del PIA con el liderazgo del físico Conrado Varotto consideraron conveniente constituir una empresa, bajo la figura legal de Sociedad del Estado (Thomas, Versino y Lalouf, 2004). Nace así, en 1976, la empresa INVAP S.E. (Investigación Aplicada - Sociedad del Estado) constituida a partir de un acuerdo entre la CNEA y la provincia de Río Negro, con el objetivo central de apoyar el Plan Nuclear argentino y crear fuentes de trabajo genuinas en la provincia.

En 1978, INVAP S.E se asocia a la CNEA para diseñar y construir una planta de enriquecimiento de uranio en la localidad de Pilcaniyeu (Río Negro). Se pretendía sustituir el uranio natural como combustible en las centrales que el país tendría en funcionamiento. Cabe mencionar que la mayoría de los reactores nucleares que hay en el mundo necesitan uranio enriquecido a menos del $5 \%$. Este tipo de enriquecimiento permitiría una importante reducción de costos por menor uso de combustible.

En amplios sectores de la comunidad científica-tecnológica este proyecto fue considerado el objetivo tecnológico más ambicioso de nuestro país. Se trata de un tema sensible internacionalmente, dado que el uranio enriquecido puede ser utilizado como combustible para las centrales nu- cleares o para armamento nuclear. Solo muy pocos países cuentan con esta tecnología, y dado su carácter estratégico, prácticamente no existen estudios publicados, ni congresos ni seminarios científicos que señalen los caminos para desarrollar esta tecnología. Contar con la capacidad tecnológica de enriquecer uranio significa generar un camino autónomo para el funcionamiento de nuestras plantas nucleares al no requerir la importación de combustible nuclear.

Este proyecto fue liderado por Conrado Varotto, y se llevó a cabo en la más absoluta reserva en el complejo tecnológico Pilcaniyeu. En su desarrollo se llevó a cabo un extenso trabajo de experimentación para la obtención de los diferentes materiales y la fabricación de los componentes de la planta, alcanzando en 1983, mediante el proceso de difusión gaseosa, la tecnología de enriquecimiento de uranio.

Los ciclos neoliberales en el país impusieron importantes obstáculos al desarrollo autónomo, de modo que como sucedió con muchos otros proyectos de desarrollo tecnológico, la planta de Pilcaniyeu sufrió en los '90 un proceso de desinversión y abandono. En el año 2007, bajo el relanzamiento del Plan Nuclear Argentino, la planta pasó de un plantel de 20 personas a más de 120 y se lograron importantes avances tecnológicos tales como la construcción de un módulo experimental de difusión gaseosa para enriquecer uranio y el reinicio de la planta piloto de enriquecimiento. Lamentablemente, una vez más, la planta quedó fuera de operaciones durante el nuevo período neoliberal 2016-2019. Actualmente, en el presupuesto nacional de 2021 está proyectado relanzar el Complejo Pilcaniyeu, contando para tal fin con un monto de $\$ 134.912 .000$, aproximadamente 1.700 .000 dólares estadounidenses, para la puesta en marcha del módulo de enriquecimiento de uranio. Por otro lado, se ha propuesto consolidar la capacidad tecnológica nacional de enriquecimiento de uranio mediante tecnologías 
| Ciencia, Tecnología y Política | Año 4 | N7 | e068 | Noviembre 2021 | ISSN 2618-3188 | www.revistas.unlp.edu.ar/CTyP |

más modernas que la difusión gaseosa, como el enriquecimiento por láser y la centrifugación.

A pesar de la importancia de este proyecto hay que tener en cuenta que el enriquecimiento de uranio en el Centro Tecnológico Pilcaniyeu se realiza a escala experimental y no a escala industrial, por lo que no alcanza para abastecer de combustible a las centrales nucleares nacionales. Llevar la planta a escala industrial requeriría de un esfuerzo continuado de varios años. Sin embargo, sería un hito importantísimo para que el país obtenga definitivamente el dominio completo del ciclo de combustible nuclear. De no hacerlo, se corre el riesgo de limitar seriamente el desarrollo nuclear nacional y el acceso a un mercado de tecnología muy promisorio y rentable dominado por muy pocos.

Desde su creación INVAP S.E. también se destacó en el diseño y construcción de reactores de investigación tanto en el país como en el extranjero. Además, sus capacidades tecnológicas acumuladas le permitieron incursionar con éxito en los campos de la medicina nuclear, las energías renovables y la tecnología satelital.

\section{EI proyecto CAREM y el futuro nu- cleoeléctrico argentino}

El proyecto CAREM (Central Argentina de Elementos Modulares) se propone desarrollar un reactor nuclear modular de baja potencia de diseño y construcción nacional. Este proyecto es producto de un largo recorrido que no estuvo exento de dificultades y de notables retrasos. Sus inicios se remontan a 1984 cuando se presenta la idea en una conferencia de la Organización Internacional de Energía Atómica, en la ciudad de Lima. Al año siguiente CNEA, en vinculación con INVAP S.E., da comienzo al proyecto CAREM. Más de diez años después, en 1997, a pedido de la CNEA, la empresa INVAP S.E. construye el reactor argentino RA-8 con la única misión de testear el núcleo del reactor CAREM. El RA-8 se construyó en Pilcaniyeu, y funcionó hasta el 2001. Ese tipo de reactores suele tener baja potencia y vida operativa breve, ya que sale de servicio una vez cumplida su función de probar la calidad de los elementos combustibles y el diseño del núcleo en un modelo físico a escala real. En febrero del 2014 se da comienzo a la construcción civil del primer prototipo CAREM 25 (de 25 MWe de potencia) en la localidad de Lima, provincia de Buenos Aires. De esta forma CAREM se constituyó en uno de los primeros reactores modulares de baja potencia del mundo en estar oficialmente en construcción, demostrando la capacidad autónoma del país para desarrollar este tipo de tecnología. El CAREM presenta innovaciones tecnológicas que simplifican enormemente su construcción, operación y mantenimiento. Por ejemplo, sus sistemas pasivos de seguridad se basan en la ley de la gravedad, y la integración del circuito primario, parte del circuito secundario y los mecanismos de control están en un solo recipiente auto-presurizado. Esto elimina la necesidad de bombas y otras instalaciones externas, como así también la cantidad y tamaño de las cañerías, reduciendo así la posibilidad de pérdida de refrigerante. Esta simplicidad conceptual es la base de su seguridad inherente que califica al sistema como de tercera generación plus.

El prototipo CAREM 25 es también una primera versión de un camino para construir reactores comerciales de exportación que puedan alcanzar una potencia entre 100 y 120 MW. En paralelo con la construcción del primer prototipo, la CNEA avanza con el diseño conceptual de los futuros CAREM comerciales, con el objetivo de generar una plataforma central multi-reactor que permitirá alcanzar costos muy competitivos en el mercado internacional. La CNEA estima que el mercado mundial de SMR asciende a unos 400.000 millones de dólares y que Argentina podría obtener un 15\% de ese mercado, o sea 60.000 millones de dólares, lo que representaría una enorme can- 
tidad de divisas para el país, al mismo tiempo que potenciaría enormemente la trama productiva nacional vinculada a la industria nuclear.

Originalmente estaba planificado que CAREM 25 se terminase en 2019, pero debido a la limitación de recursos durante el ciclo neo-liberal 2016-2019 se estima que su concreción será en el año 2023. La administración nacional que asumió a fines de 2019 reimpulsó el plan nuclear, tal cual se refleja en el presupuesto nacional 2021. En el mismo se dispone de casi $\$ 18.500$ millones con el objetivo de recuperar y sostener al sector nuclear nacional. En este presupuesto se describe a la construcción de la Central Nuclear CAREM 25 como uno de los grandes proyectos estratégicos, y se le asignan \$6.177 millones de pesos, equivalente a un tercio del presupuesto total del sector nuclear.

La proyección del sector nuclear nacional no puede estar supeditada únicamente a la construcción del CAREM, pues como todo gran desarrollo tecnológico es un camino experimental que suele tener imprevistos. Es por ello que también se está avanzado en otros proyectos nucleares que refuerzan las señales de continuidad en el desarrollo del sector. En este sentido se han retomado las conversaciones con China para la construcción de futuras centrales nucleares, para lo cual se había realizado un convenio en 2015, que fue posteriormente disuelto durante el gobierno de Mauricio Macri. En esa ocasión se acordó construir dos centrales, las futuras cuarta y quinta centrales nucleares del país, la primera de estas a base de uranio enriquecido y la segunda a base de uranio natural. Para este proyecto se había creado un consorcio entre NA-SA ${ }^{1}$ y la Compañía Nacional Nuclear China, en miras a que tecnólogos de ambos países trabajaran en ambos proyectos. Actualmente, se proyecta empezar con la obra de la cuarta central nuclear cuya construcción comenzaría en junio de 2022, pues la misma ya cuenta con el financiamiento que proviene de China. Esta futura central, Ilamada Atucha III, se basa en la tecnología Hualong de uranio enriquecido, razón por lo cual la participación de la industria nacional va a estar limitada, sobre todo en los rubros metalmecánico, químico y eléctrico. Sin embargo, desde el sector nuclear nacional se están negociando algunas cláusulas contractuales para favorecer la incorporación de tecnología local, en particular para que haya transferencia de tecnología a la CNEA para la fabricación del combustible nuclear de esta planta. La quinta central nuclear se estima que se iniciará en 2024 y sería a base de uranio natural y agua pesada. También se pensó un cambio en su futuro cronograma de construcción, realizando la ingeniería del proyecto y comenzando con la compra de los componentes a adquirir localmente, mucho antes de empezar la obra civil. Se prevé que esta central generará una demanda significativa en la industria local asociada al sector nuclear.

Por otra parte, actualmente hay otras dos obras de envergadura que son la extensión de la vida útil de Atucha I y II, y el Almacenamiento en Seco de los Elementos Combustibles Quemados (ASECQ I y II).

\section{Capacidades y recursos del complejo nuclear argentino}

La tabla 1 muestra sintéticamente los principales activos que componen el sector nuclear argentino y áreas que utilizan este tipo de tecnología (Comisión Nacional de Energía Atómica, 2015 y 2016).

\footnotetext{
${ }^{1}$ NA-SA es el acrónimo de la empresa Nucleoeléctrica Argentina S.A. y está a cargo de la operación de las centrales nucleares del país, fue creada en 1994, y su participación accionaria se divide entre el Ministerio de Economía de la Nación con 79\%, la Comisión Nacional de Energía Atómica con el 20\% e Integración Energética Argentina S.A. (IEASA) con el 1\%.
} 


\begin{tabular}{|c|c|c|c|c|c|c|}
\hline \multicolumn{7}{|c|}{ Actividades e Instalaciones Bajo Control o Regulación Estatal Federal } \\
\hline Actividad / Instalación & Nombre & Sigla & Locación & Aplicación & Pertenencia & Referencia \\
\hline \multirow{3}{*}{ Centros Atómicos } & Centro Atómico Bariloche & $\mathrm{CAB}$ & Río Negro & \multirow{3}{*}{\begin{tabular}{|l|} 
Investigación científica de base \\
hasta la elaboración de soluciones \\
tecnológicas de alto valor \\
agregado
\end{tabular}} & Estado Nacional & 1955 - Creación \\
\hline & $\begin{array}{l}\text { Centro Atómico } \\
\text { Constituyentes }\end{array}$ & CAC & Bs. As. & & Estado Nacional & 1958 - Creación \\
\hline & Centro Atómico Ezeiza & CAE & Bs. As. & & Estado Nacional & 1967 - Creación \\
\hline \multirow{3}{*}{$\begin{array}{l}\text { Centrales Nucleares de Potencia en } \\
\text { Operación }\end{array}$} & Central Nuclear Atucha I & CNA-I & Bs.As. & Generación de Energía Eléctrica & Estado Nacional & $\begin{array}{c}1974 \text { - Comienzo de } \\
\text { operación }\end{array}$ \\
\hline & Central Nuclear Atucha II & CNA-II & Bs.As. & Generación de Energía Eléctrica & Estado Nacional & $\begin{array}{l}2014 \text { - Comienzo de } \\
\text { operación }\end{array}$ \\
\hline & Central Nuclear Embalse & CNE & Bs.As. & Generación de Energía Eléctrica & Estado Nacional & $\begin{array}{c}1984 \text { - Comienzo de } \\
\text { operación }\end{array}$ \\
\hline $\begin{array}{l}\text { Central Argentina de Reactores de } \\
\text { Elementos Modulares (En } \\
\text { construcción) }\end{array}$ & CAREM & CAREM 25 & Bs.As. & Generación de Energía Eléctrica & Estado Nacional & $\begin{array}{l}2014 \text { - Comienzo de la } \\
\text { construcción }\end{array}$ \\
\hline Planta de Enriquecimiento de Uranio & $\begin{array}{l}\text { Complejo Tecnológico } \\
\text { Pilcaniyeu }\end{array}$ & СTP & Río Negro & Producción de Uranio enriquecido & Estado Nacional & 1984 - Inauguración \\
\hline Planta Industrial de Agua Pesada & $\begin{array}{l}\text { ENSI (Empresa CNEA - Estado } \\
\text { Prov. Neuquén, que opera la } \\
\text { planta) }\end{array}$ & PIAP & Neuquén & Producción de agua pesada & $\begin{array}{l}\text { CNEA - Estado } \\
\text { prov Neuquén }\end{array}$ & $\begin{array}{l}1990 \text {-Comienzo de } \\
\text { operación }\end{array}$ \\
\hline Planta de purificación de Uranio & \begin{tabular}{|l|} 
Dioxitek (Consorcio con \\
participación de Sec. De \\
Energia de la Nación, CNEA \\
y Prov. De Mdza.) \\
\end{tabular} & PPU & Córdoba & $\begin{array}{l}\text { Producción de Dióxido de Uranio } \\
\text { para combustible nuclear, Fuentes } \\
\text { selladas de Cobalto-60 para rayos } \\
\text { gamma y Molibdeno } 99 \text { para } \\
\text { radiofármacos }\end{array}$ & $\begin{array}{l}\text { Sec. De Energía - } \\
\text { CNEA - Estado } \\
\text { Provincial Mdza }\end{array}$ & 1982 - Creación \\
\hline $\begin{array}{l}\text { Nueva planta de purificación de } \\
\text { Uranio (en construcción) }\end{array}$ & $\begin{array}{l}\text { Dioxitek (Consorcio con } \\
\text { participación de Sec. De } \\
\text { Energia de la Nación, CNEA } \\
\text { y Prov. De Mdza.) } \\
\end{array}$ & NPUO2 & Formosa & $\begin{array}{l}\text { Abastecimiento de combustible } \\
\text { para centrales nucleares (UO2) }\end{array}$ & $\begin{array}{l}\text { Sec. De Energia - } \\
\text { CNEA - Estado } \\
\text { Provincial Mdza }\end{array}$ & $\begin{array}{l}2014 \text { - Creación- En } \\
\text { construcción }\end{array}$ \\
\hline $\begin{array}{l}\text { Reactores Experimentales y de } \\
\text { Producción de Radioisótopos }\end{array}$ & REP & $\begin{array}{l}\text { RA-1, RA-0, RA- } \\
\text { 3, RA-4, RA-6, } \\
\text { RA-8. \# RA-10 } \\
\text { (construcción) }\end{array}$ & $\begin{array}{l}\text { Córdoba, Sta } \\
\text { Fe, Bs. As., } \\
\text { R. Negro }\end{array}$ & $\begin{array}{l}\text { Investigación, Desarrollo, } \\
\text { Docencia }\end{array}$ & Estado Nacional & $\begin{array}{c}\text { 1957, 1965, 1967, 1972, } \\
\text { 1982, 1997. Año } 2014 \\
\text { CNEA obtiene la licencia } \\
\text { para la construcción del } \\
\text { RA-10 }\end{array}$ \\
\hline \multirow{4}{*}{ Institutos de Formación Académica } & Instituto Balseiro & IB & \begin{tabular}{|l} 
Río Negro - \\
CAB \\
\end{tabular} & $\begin{array}{l}\text { Formación - Carreras de grado y } \\
\text { posgrado }\end{array}$ & Estado Nacional & 1955 - Fundación \\
\hline & $\begin{array}{l}\text { Instituto de Tecnología } \\
\text { Nuclear Dan Beninson }\end{array}$ & IDB & Bs.As.- CAE & $\begin{array}{l}\text { Formación, especialización nuclear } \\
\text { en el ámbito de la salud - Carreras } \\
\text { desde Pregrado hasta Doctorados }\end{array}$ & $\begin{array}{l}\text { Estado Nacional } \\
\text { (CNEA-UNSAM) }\end{array}$ & 2006 - Creación \\
\hline & Instituto Sabato & is & Bs. As. - CAC & $\begin{array}{l}\text { Formación relacionada con el } \\
\text { estudio de los materiales- Carreras } \\
\text { de grado y posgrado }\end{array}$ & $\begin{array}{l}\text { Estado Nacional } \\
\text { (CNEA-UNSAM) }\end{array}$ & 1993 - Creación \\
\hline & $\begin{array}{l}\text { Fundación Escuela de } \\
\text { Medicina Nuclear en la } \\
\text { ciudad de Mendoza }\end{array}$ & FUESMEN & Mendoza & $\begin{array}{l}\text { La FUESMEN desarrolla } \\
\text { actividades docentes de pre y pos } \\
\text { grado en medicina nuclear y } \\
\text { radiodiagnóstico }\end{array}$ & $\begin{array}{l}\text { CNEA-UNC- } \\
\text { Estado } \\
\text { Provincial }\end{array}$ & 1991 - Creación \\
\hline \multirow{7}{*}{ Centros de Medicina Nuclear } & $\begin{array}{l}\text { Centro de Medicina Nuclear } \\
\text { del Hospital de Clínicas José } \\
\text { de San Martín }\end{array}$ & $\mathrm{CMN}$ & Bs. As. & Medicina nuclear & CNEA-UBA & $\begin{array}{c}1959 \text { Inauguración Lab. } \\
\text { Radioisótopos, } 1969 \\
\text { comienzo de operación } \\
\text { CMN }\end{array}$ \\
\hline & $\begin{array}{l}\text { Centro Oncológico de } \\
\text { Medicina Nuclear del } \\
\text { Instituto Oncológico Ángel H. } \\
\text { Roffo (COMNIR) }\end{array}$ & COMNIR & Bs.As. & $\begin{array}{l}\text { Diagnóstico por imágenes, } \\
\text { Medicina nuclear, Terapia radiante }\end{array}$ & CNEA- UBA & 1969 - Creación \\
\hline & $\begin{array}{l}\text { Fundación Escuela de } \\
\text { Medicina Nuclear en la } \\
\text { ciudad de Mendoza } \\
\text { (FUESMEN) }\end{array}$ & FUESMEN & Mendoza & $\begin{array}{l}\text { PET/CT y PET/MR, Cámara Gamma } \\
\text { SPECT, Servicios Profesionales de } \\
\text { Radiofísica Sanitaria, Tomografia, } \\
\text { Densitometría, Otros }\end{array}$ & $\begin{array}{l}\text { CNEA-UNC- } \\
\text { Estado } \\
\text { Provincial }\end{array}$ & 1991 - Inauguración \\
\hline & \begin{tabular}{|l|} 
Centro de Diagnóstico \\
Nuclear en la Ciudad \\
Autónoma de Buenos Aires
\end{tabular} & FCDN & Bs. As. & $\begin{array}{l}\text { PET/CT, Tomografía multicorte, } \\
\text { Medicina nuclear -SPECT-CT }\end{array}$ & $\begin{array}{l}\text { CNEA- UNC- } \\
\text { Estado } \\
\text { Provincial }\end{array}$ & 2004 - Creación \\
\hline & \begin{tabular}{|l}
$\begin{array}{l}\text { Centro de Medicina Nuclear } \\
\text { de Entre Ríos }\end{array}$ \\
\end{tabular} & CEMENER & Entre Ríos & $\begin{array}{l}\text { Quimioterapia, Radioterapia, } \\
\text { Diagnóstico por imagenes }\end{array}$ & $\begin{array}{l}\text { CNEA- Estado } \\
\text { Provincial }\end{array}$ & 2015 - Creación \\
\hline & \begin{tabular}{|l|} 
Instituto de Tecnologías \\
Nucleares para la Salud \\
\end{tabular} & INTECNUS & Rio Negro & $\begin{array}{l}\text { Quimioterapia, Radioterapia, } \\
\text { Diagnóstico por imagenes }\end{array}$ & $\begin{array}{l}\text { CNEA- Estado } \\
\text { Provincial }\end{array}$ & $\begin{array}{c}\text { 2019- Inauguración de } \\
\text { CMN }\end{array}$ \\
\hline & $\begin{array}{l}\text { Centro de Medicina Nuclear } \\
\text { de la Patagonia Austral }\end{array}$ & CEMNPA & Santa Cruz & $\begin{array}{l}\text { Medicina nuclear, Quimioterapia. } \\
\text { Radioterapia }\end{array}$ & $\begin{array}{c}\text { CNEA- Estado } \\
\text { Provincial }\end{array}$ & 2018-Creación \\
\hline Complejo Minero Fabril & $\begin{array}{l}\text { Complejo Minero Fabril San } \\
\text { Rafael }\end{array}$ & CMFSR & Mendoza & Planta de concentración de Uranio & $\begin{array}{l}\text { CNEA- Estado } \\
\text { Provincial } \\
\end{array}$ & 1979 - Inauguración \\
\hline Planta de irradiación semi-industrial & $\begin{array}{l}\text { Planta de irradiación semi- } \\
\text { industrial }\end{array}$ & PISI & CAE - Bs. As. & $\begin{array}{l}\text { Irradiación de muestras y } \\
\text { productos }\end{array}$ & CNEA & 1970- Construcción \\
\hline Proyecto PIPAE & $\begin{array}{l}\text { Planta de Irradiación por } \\
\text { aceleración de electrones }\end{array}$ & PIPAE & CAE - Bs. As. & $\begin{array}{l}\text { Producción de Rayos } X \text { de alta } \\
\text { energía. Investigación y } \\
\text { experimentación }\end{array}$ & CNEA & $\begin{array}{l}\text { 2016- Llamado a } \\
\text { licitación para } \\
\text { construcción } \\
\end{array}$ \\
\hline Acelerador de iones pesados. & $\begin{array}{l}\text { Acelerador Tandem } \\
\text { Argentino }\end{array}$ & TANDAR & CAC-Bs. As. & Investigación & CNEA & 1985 - Inauguración \\
\hline Acelerador de partículas & $\begin{array}{l}\text { Ciclotrón de producción de } \\
\text { radioisótopos }\end{array}$ & - & CAE - Bs. As. & Producción de radioisótopos & CNEA & 1994- Inauguración \\
\hline
\end{tabular}


La columna "Pertenencia" de la Tabla I muestra cómo diferentes instancias del Estado Nacional son las que actúan como partes principales del grupo de "propietarios" en representación del sector público, en las actividades, capacidades, instalaciones y recursos del complejo nuclear argentino.

\section{Conclusiones}

Argentina se propuso llevar adelante una política nuclear y un desarrollo tecnológico con fines pacíficos, empezando con una primera etapa de capacitación de su personal, y luego mediante la construcción de reactores de investigación, y la producción de combustible nuclear para su operación. A fines de la década del sesenta centró sus recursos en la generación de energía eléctrica, comenzando la etapa de construcción de centrales nucleares y el dominio del ciclo de combustible.

Todos estos logros fueron producto de una política de Estado que tuvo como sustento una virtuosa alianza entre científicos y tecnólogos destacados y distintos gobiernos. Esta interacción se mantuvo hasta 1983, permitiendo el logro de varios de los objetivos parciales del Plan Nuclear, y con ello el posicionamiento de liderazgo de Argentina en la región.

A partir de 1983 comenzaron los recortes presupuestarios y posteriormente en la década de los noventa, con la llegada al poder de un gobierno neoliberal, se produjo una paralización y desmantelamiento de las capacidades instaladas. Luego de esta etapa que no sólo perjudicó al sector nuclear sino a la industria nacional en general, el gobierno de Néstor Kirchner reedita el Plan Nuclear dando un fuerte impulso al área.

A pesar de este recorrido con idas y vueltas, el desarrollo nuclear de la Argentina ha tenido hitos destacados. Entre ellos la obtención de uranio enriquecido en la Planta de Pilcaniyeu, desarrollo que fue realizado íntegramente por tecnólogos nacionales, la creación de INVAP S.E., un spin-off de CNEA, que se ha destacado por su rol en el diseño y construcción de reactores de investigación y en el campo de la medicina nuclear. A lo que se le suma el Proyecto CAREM que tiene una gran potencialidad, no solo para la política energética de nuestro país, sino por la posibilidad de transferir tecnología hacía países demandantes de centrales nucleares de baja potencia.

\section{Bibliografía}

Bisang, R. (1995). Libre mercado, intervenciones estatales e instituciones de Ciencia y Tecnología en la Argentina: apuntes para una discusión. Redes, 2(3), 13-58.

Colombo, S., Guglielminotti, C., y Nevia Vera, M. (Enero-Junio de 2017). El desarrollo nuclear de Argentina y el régimen de no proliferación. Perfiles Latinoamericanos, 49, 1-21.

Comisión Nacional de Energía Atómica (2015). Plan Estratégico 2015-2025. https://www.cnea. gob.ar/nuclea/handle/10665/971

Comisión Nacional de Energía Atómica (2016). Memoria y Balance 2016. https://www.cnea.gob. ar/nuclea/handle/10665/1016

Consejo Argentino para las Relaciones Internacionales (1999). La Argentina exportadora de tecnología nuclear. http://www.cari.org.ar/pdf/nuclearesp. pdf

De Dicco, R. (Junio de 2015). Breve historia de la Central Nuclear Atucha II, 1974 - 2015. Observatorio de la Energía, Tecnología e Infraestructura del Desarrollo. https://www.oetec.org/informes/atuchaii050615.pdf

Hurtado de Mendoza, D (Enero de 2005). De "átomos para la paz" a los reactores de potencia. Tecnología y política nuclear en la Argentina (1955- 
1976). Revista Iberoamericano de Ciencia, Tecnología y Sociedad - CTS, 2(4), 41-66.

Hurtado de Mendoza, D. (Septiembre de 2009). Periferias y Fronteras Tecnológicas. Energía Nuclear y dictadura militar en Argentina (1976-1983). Iberoamericano de Ciencia, Tecnología y Sociedad - CTS, 5(13), 27-64.

Hurtado de Mendoza, D (2014). El Sueño de la Argentina Atómica. Política, tecnología nuclear y desarrollo nacional (1945-2006). Edhasa.

Nevia Vera, M. y Colombo, S. (Enero de 2014). La política nuclear argentina y la cooperación estratégica con Brasil en el siglo XXI. Intellector, XI(21), 16-29.

Quilici, D. (2008). Desarrollo de proveedores para la industria nuclear argentina. Visión desde las Centrales Nucleares. H-industri@: Revista de historia de la industria argentina y latinoamericana, 2(2).

Quilici, D. (Enero/Junio de 2010). La fabricación de los elementos combustibles para los reactores nucleares de potencia en Argentina: Un caso de inversiones productivas realizadas por un organismo de ciencia y técnica. CNEA, 10(37-38), 2339.

Sábato, J. (Octubre/Diciembre de 1968). Energía Atómica en Argentina. Estudios Internacionales, 2(3), 332-357.

Thomas, H., Versino, M., Lalouf, A. (2004). Producción de bienes conocimiento-intensivos en países subdesarrollados. Trayectoria socio-técnica de una empresa nuclear y espacial argentina. VI Jornadas de Sociología, Universidad de Buenos Aires. Facultad de Ciencias Sociales. https://cdsa. aacademica.org/000-045/34.pdf 